Journal of Computer Science 8 (7): 1156-1164, 2012

ISSN 1549-3636

(C) 2012 Science Publications

\title{
An Optimal Rate Adjustment Algorithm for Medium Access Control Protocol in 802.11 Multi Hop Ad Hoc Networks
}

\author{
${ }^{1}$ K. Saravanan and ${ }^{2} \mathrm{~T}$. Ravichandran \\ Anna University of Technology-Coimbatore, Tamilnadu, India
}

\begin{abstract}
Problem statement: Medium Access Control (MAC) protocol plays an important role in providing a fair and efficient allocation of limited bandwidth in wireless LANs. In IEEE 802.11 standard protocol, data rate selection is not specified. Rate control is the process of switching data rates dynamically based on channel conditions, with the target of selecting the rate that will provide the maximum throughput feasible for a given channel condition. The two major components of the rate control process are Channel estimation and rate selection. Although rate control has been studied extensively for wired networks, these results cannot be directly applied to multihop wireless networks. Approach: In this study, we propose to develop an Optimal Rate Adjustment Algorithm (ORAA) which is based on the channel state conditions. We also follow a two level channel estimation one at the receiver end and another at each intermediate node along the path. Results: By simulation results we show that our proposed ORAA achieves high throughput and fairness, when compared with the standard IEEE 802.11 MAC protocol. Conclusion: In ad hoc networks, where the channel conditions are dynamic, our proposed ORAA provides the accurate data rate most suitable for the current changes in the network.
\end{abstract}

Key words: Medium Access Control (MAC), Optimal Rate Adjustment Algorithm (ORAA), IEEE 802.11, Signal to Noise Ratio (SNR)

\section{INTRODUCTION}

Multi hop ad hoc networks: A set of nodes that can communicate with each other devoid of well-known infrastructure or centralized control is termed as multihop wireless ad hoc networks. In this network, every node represents the wireless transceiver that performs the transmission and reception which is common to all nodes at a single frequency band. Though they are restricted by their transmitting and receiving capacity, the nodes can communicate with each other. Hence they cannot reach the entire nodes in the network directly since the majority of the nodes are outside of direct range. Under this scenario, the transmission of information between two nodes is made possible using other nodes in the network. To overcome these issues, the network function in multihop fashion. The nodes are involved in routing the traffic for each other. Hence a packet can travel from any source to its destination directly or via some number of intermediate packet forwarding nodes.

The process of forcing more traffic into the networks may result in a high packet loss rate, rerouting instability and unfairness issues in multi-hop ad hoc networks. When the traffic was forwarded at the time of traffic flow among source and destination in a multi - hop network, the nodes in the middle of the path should handle additional nodes. The source node forced more traffic into the path at the time of lighter contention when compared to the nodes that forward the traffic later. This can further result in excessive packet losses and re-routing instability. In the scenario where some flows existing in the multiple flows experience higher contention when compared to other flows, causes inequality. (Shrivastava and Sahu, 2011).

IEEE 802.11 standards: IEEE 802.11 plays a major role in the next generation of wireless and mobile communication systems. IEEE 802.11 Direct Sequence Spread Spectrum (DSSS) provided just two physical data rates at the beginning. And every transmission was performed at $1 \mathrm{Mbps}$ or $2 \mathrm{Mbps}$ rate. In 1999, the IEEE defined two high rate extensions:

- Based on DSSS technology, 802.11b is defined with data rates up to $11 \mathrm{Mbps}$ in the $2.4 \mathrm{GHz}$ band

- Based on Orthogonal Frequency Division Multiplexing (OFDM) technology, 802.11a is defined with data rates up to $54 \mathrm{Mbps}$ in the $5 \mathrm{GHz}$ band

In 2003, the 802.11g standard was confirmed that is the extended version of $802.11 \mathrm{~b}$ PHY layer for

Corresponding Author: Saravanan, K., Anna University of Technology - Coimbatore, Tamilnadu, India 


\section{J. Computer Sci., 8 (7): 1156-1164, 2012}

facilitating data rates up to $54 \mathrm{Mbps}$ in the $2.4 \mathrm{GHz}$ band. [method3].

Rate control in ad hoc networks: The process of dynamically switching data rates based on channel conditions for selecting the rate that will offer maximum throughput is termed as rate control. This mechanism has been shown to improve the performance of wireless networks, which suffer from fading and interference. Channel estimation and the rate selection can be considered as the two major components of the rate adaptation process.

The channel quality estimation is engaged in measuring the channel condition with respect to time in order to generate the future quality predictions. The issues concerned with this estimation is that the metrics related to channel quality indication and prediction method whether short or long-term are not specified.

Hence following two issues are essential in the channel estimation process:

- Identifying metrics to be used as indicators of channel quality. For instance SNR, signal strength, symbol error rate, BER

- Algorithms to be used for channel prediction

The rate selection utilizes the channel quality predictions to select suitable rate. In general, the threshold selection technique is utilized for rate selection. In this technique, the value of an indicator is compared with a threshold value list that indicates the boundaries among the data rates. In practice data transmission rates can be varied by different modulation schemes and/or coding techniques. Modulation is the process of translating an outgoing data stream into a form suitable for transmission on the channel. It involves transforming the data stream into a sequence of symbols. Each symbol may encode a number of bits depending on the modulation scheme used. The symbol sequence is then transmitted at a certain rate, the symbol rate, such that the data rate is determined by the number of encoded bits per symbol for a given symbol [journal, mobicom]. It is not possible to apply these results in multihop wireless networks, though the rate control has been widely studied for wired networks. Each link capacity is fixed in wired networks. But the link capacity varies at each time in wireless networks. The rate control technique proposed in the past took either single-hop flows into consideration or enforces basic assumption on a limited number of scheduling policies. Therefore, these works have not utilized the advantage of multihop communication and joint multi-layer control completely.
The effectiveness of rate adaptation depends on the accuracy of the channel quality estimates. Furthermore, once good estimates are generated, it is important to use them before they become outdated. Therefore, it is also advantageous to minimize the delay between the time of the channel estimate and the time the packet is transmitted with the selected data rate [journal, mobicom].

Rate control in medium access control and its types: The multi rate features are provided by the physical layer of the protocol architecture. To exploit the full potential of multi rate transmissions, MAC layer of the protocol architecture should also be adapted to different transmission rates. A number of rate adaptive MAC layers suited to 802.11 multi rate physical layers have been proposed in the last decade [journal].

Auto Rate Fallback (ARF): ARF was the first commercial 802.11 based MAC layer that supports the multiple transmission rates. It was mainly intended to improve the application throughput which in turn resulted in the 802.11 DSSS standard. Following a set of successful transmissions at a specified rate, every sender in ARF tries to utilize higher transmission rate. Similarly, following one or two successive failures, sender shifts to lower rates. In case of two successive transmission failures in a row, this algorithm minimizes the current rate and initiates the timer. Whereas the timer is reset and transmission rate is enhanced when timer expires or set of successfully received per packet acknowledgments attains value 10. Thus at the time of increase in rate, the primary transmission following the rate increase should succeed or else the rate is directly decreased and the timer is restarted rather than trying the higher rate a second time. This technique results in two issues.

The rapid changes in channel conditions is not adaptable in an efficient manner. For example, The maximum changes in rate occur in packet transmission from one packet to another in ad hoc network in which the interference bursts are generated by another 802.11 packet transmission. Since the algorithm necessitates one or two packet failures to reduce its rate and maximum of 10 successful packet transmissions for rate enhancement, it is difficult to synchronize it with the sub-packet channel condition changes.

In case there are nil or slight channel condition changes, it attempts to utilize a higher rate for each 10 successful packet transmissions. This in turn maximizes the retransmissions activity and thereby decreasing the application throughput. 


\section{J. Computer Sci., 8 (7): 1156-1164, 2012}

RBAR: This is the only alternative algorithm for rate adaptation to improve the application throughput. This algorithm necessitates alterations in IEEE 802.11 standard. The understanding of certain MAC control frames is altered and every data frame should contain a new header field. This algorithm allows the utilization of RTS/CTS techniques. In prior to initiation of every data transmission, a pair of request termed as to send and clear to send control frames are swapped among the source and destination nodes. Depending upon the received RTS frame's Signal To Noise Ratio (SNR) and a priori wireless channel model based computation of a set of SNR thresholds, the RTS frame computes the transmission rate to be utilized by the upcoming data frame transmission. Later, the rate to be utilized is then forwarded to the source in the CTS packet.

The rate to be utilized is feedback to source using CTS packet. For updating the Network Allocation Vector (NAV) exactly, the RTS, CTS and data frames are altered to hold the size and data transmission rate information for permitting every node within the transmission range. The issues concerned with this protocol are as follows.

- Each receiver selects an optimal feasible rate using a threshold mechanism that necessitates a computation of SNR thresholds according to priori channel model

- The assumption that the availability of SNR of a given packet at the receiver is false

- It necessitates the RTS/CTS protocol although hidden nodes are absent

- The RTS and CTS frames interpretation and data frames format does not match with the 802.11 standard

Opportunistic Auto Rate (OAR): OAR algorithm helps in utilizing high quality channels during the transmission of multiple back-to-back packets. Specifically, following the indication of optimal channel quality by the multi rate MAC, this algorithm allows channel access for multiple packet transmissions. As a result, OAR nodes transmit more packets under high quality channels when compared to low quality channels. But OAR cannot randomly support flows with optimal channel quality, since flows access to everlasting bad channels need to be guaranteed. This algorithm also makes sure that entire flows are allocated with a similar temporal share of channel access. OAR can offer various throughputs for flows, according to their channel conditions but all flows can attain about identical time shares. The demerits of this approach are:
- It needs a multi rate MAC protocol namely RBAR or ARF for medium access at rates above the base rate though it is applicable to both sender and receiver based protocols

- It needs a mechanism contain the channel for an extended packet transmission set during the provision of a high rate channel by RBAR. (Hieu and Hong, 2010)

Metrics to be analyzed: The following are the performance metrics that need to be considered at the time of MAC protocol evaluation for wireless mobile ad hoc network.

Throughput: It is the measure of successfully transmitted radio link level frames per unit time.

Transmission delay: The time interval between the frame arrival time of the transmitter's MAC layer and the time at which the transmitter recognizes that the transmitted frame is received by the receiver successfully is referred to as transmission delay.

Fairness: It represents the way by which the channel is allocated among the flows in various mobile nodes. The two major factors are influencing the fairness are node mobility and unreliability of radio channels.

Energy efficiency: It is the ratio of useful energy consumption to the total energy expenditure (Pillai et al., 2010).

Packet error rate: The packet error rate is generally calculated by the Bit Error Rate (BER) and the packet length. The receiver estimates the SNR for the RTS packet and gets the BER with a transmission rate by the equations derived from an analytical model (Nguyen $e t$ al., 2011).

In our previous work, we have proposed a cross layer based MAC protocol for utilizing the channel bandwidth absolutely and enhancing flow fairness deviod of congestion. We used a probing technique to estimate the available bandwidth along each path of the source and destination pair. Then paths with adequate bandwidth and least contention is selected by the source with the help of a multipath routing protocol. Besides this, to overcome the overheads and issues of IEEE 802.11, the centralized flow scheduler was designed. This scheduler schedules the flows as an alternative of nodes. As an extension to our previous work, we propose an optimal rate control mechanism for the MAC protocol in 802.11 multi-hop ad hoc networks. 


\section{J. Computer Sci., 8 (7): 1156-1164, 2012}

Related work: Priakanth and Thangaraj (2009) proposed a channel adaptive Energy efficient Medium Access Control (MAC) protocol in an ad hoc network. In their approach, initially each node computes the channel and link quality for each contending flows. As per the computation, the weight value is computed and propagated using the routing protocol. Their protocol permits the transmission for the flows which possess weight greater than Channel Quality Threshold (CQT) since the wireless link with worse channel quality causes more expenditure of energy. They also proposed a fair scheduling and queueing algorithm for preventing buffer overflow and to achieve fairness.

Chen et al. (2007) (DRA) have proposed a Differential Rate Adaptation (DRA) for IEEE 802.11 networks. Their mechanism utilized a single RTS/CTS exchange among a given sender-receiver pair to guide multiple DATA/ACK dialogs in the sequel. Each of their ACK contained a bit to designate the sender whether there is a recommendation of next higher data rate in its header. Further, DRA facilitated a high network throughput by tuning the data transmission rate in an adaptive manner in relation to channel conditions.

Zhang et al. (2008); Chen et al. (2007); Bandai et al. (2008); Nguyen et al. (2011); Pillai et al. (2010); Sadeghi et al. (2005); Jahromizadeh (2011); Hu et al. (2011); Ashraf (2009); Nguyen and Garcia-LunaAceves (2011); Ng and Liew (2007) and Kherani et al. (2008) have conducted a regular measurement based study to confirm that SNR was a good prediction tool for channel quality and have identified two key challenges. They have found that the SNR measured in hardware has been often Uncalibrated and thus the SNR thresholds were hardware dependent. Also they have found that the direct prediction from SNR to Frame Delivery Ratio (FDR) was often over optimistic under interference conditions. Based on these observations, they have presented a novel practical SNR Guided Rate Adaptation (SGRA) scheme. Their proposed SGRA have addressed all identified challenges and was fully compliant with 802.11 standards.

Wang et al. (2007); Zhang et al. (2008); Chen et al. (2007); Bandai et al. (2008); Nguyen et al. (2011); Pillai et al. (2010); Sadeghi et al. (2005); Jahromizadeh (2011); Hu et al. (2011); Ashraf (2009) and Nguyen and Garcia-Luna-Aceves (2011) has studied the problem of using the rate adaptation technique to achieve energy efficiency in an IEEE 802.11 based multihop network. In particular they have formulated it as an optimization problem specifically minimizing the total transmission power over transmission data rates, subjected to the traffic requirements of all the nodes in a multihop network. They have followed distributed
Cooperative Rate Adaptation (CRA) for promoting node cooperation and have observed that the inequality in non cooperative channel contention among nodes caused by a hidden terminal phenomenon in a multihop network tend to result in energy inefficient. Their CRA scheme consisted of three modules, namely information exchange algorithm, rate selection algorithm and node cooperation algorithm.

Zhou et al. (2007); Wang et al. (2007); Zhang et al. (2008); Chen et al. (2007); Bandai et al. (2008); Pillai et al. (2010); Sadeghi et al. (2005); Jahromizadeh (2011); Hu et al. (2011) and Ashraf (2009) have proposed a novel scheme called Correlation based Rate Adaptation (CORA) to address the rate adjustment problem in which the transmission parameters were adjusted based on the correlation between adjustment action and results Their CORA would split the rate into more atomic components and adjusted them according to the correlation between rate adaptation actions and transmission results. They have used IEEE $802.11 \mathrm{n}$ as the context for their CORA design, where transmission mode has been expanded to spatial dimension in addition to the usual modulation and convolution coding mechanisms.

Ye et al. (2009); Zhou et al. (2007); Wang et al. (2007); Zhang et al. (2008); Chen et al. (2007); Bandai et al. (2008); Nguyen et al. (2011); Pillai et al. (2010); Jahromizadeh (2011) and $\mathrm{Hu}$ et al. (2011) have investigated the performance of IEEE 802.11 in multi hop scenarios and have shown how its aggressive behavior could throttle the spatial reuse and reduce bandwidth efficiency. They have also proposed an adaptive, layer-2 distributed coordination scheme for 802.11 using the explicit MAC feedback in order to speed the transmissions on adjacent nodes. In that way their scheme assisted the MAC protocol to operate around its saturation state while minimizing resource contention.

\section{MATERIALS AND METHODS}

Channel state estimation: The ad hoc networks is devoid of base station for functioning either as central controller or dedicated control channel to feedback the channel state. Due to these characteristics, we estimate the channel state based on the packet success rate, checked at two levels as follows:

- At the receiver end

- At each intermediate node along the path

Only if the requirements at both the levels are satisfied, the channel is confirmed to be in good condition. The 
steps for the estimation of channel state are given under Algorithm -1.

The wireless link state is estimated whether its good or bad. A packet which is transmitted on a good link contains higher success probability when compared to transmission performed over a bad link. The link conditions are independent of each other. Owing to channel errors or packet collisions, unsuccessful transmissions occur. The transmitter need not know the reason for an unsuccessful transmission.

At the receiver end: By exchanging the two short control packets between a sender and a receiver, all neighboring nodes recognize the transmission and back off during the transmission time advertised along with the RTS and CTS packets. In our channel state estimation, the CTS packets and ACK packets are checked at the receiver side. Based on the results of these packets, we classify the channels with three states namely GOOD1, BAD1 and AWAITING1. Thus, a Flag (FL) is associated to indicate the corresponding channel state. The flag can take three values: GOOD1, BAD1 or AWAITING1.

- Check for the CTS packets, which informs the sender that the packets are confirmed to be sent

- Also check for the ACK packets, which is an acknowledgement of successful data transmission

If both the above conditions are satisfied, then the channel is in GOOD state and will be checked for the subsequent conditions at the nodes. If any of the above conditions is not satisfied then the channel is in BAD state and eventually the further transmissions are dropped out.

At each intermediate node along the path: The fraction of the successful transmission count over the most recent transmissions is termed as a Packet success rate $\left(\mathrm{P}_{\mathrm{S}}\right)$. Furthermore at each node, the packet success rate $\left(P_{S}\right)$ is checked against a threshold value $\left(P_{t h}\right)$. If the value falls above the threshold value, the link is in good condition with its state marked as GOOD2 else the link is considered bad and marked as BAD2. Since the channel condition is checked at each and every node, the changes in channel are updated with the exact channel conditions.

Suppose if a path has many links with both GOOD2 and BAD2 states, then in such cases the path is valid only if it contains the maximum number of links with state GOOD2 else the path is invalid (i.e.,) not suitable for transmission and will be kept in the AWAITING2 state for a particular time period $\left(t_{t h}\right)$. For instance if there are totally 5 links in a path with 3 of the links in state GOOD2, then the path is valid as the maximum links have GOOD2 states. Suppose if only 2 of the links are in GOOD2 state, then the path is invalid. Once the channel condition improves and if the maximum number of links in the path have state GOOD2, then the path is valid. Also once the $t_{\text {th }}$ value is exceeded, then also the path is invalid and is not suitable for transmission.

\section{Algorithm -1:}

1. At the receiver,

1. 1. If CTS \&\& ACK = True, then

1. 1. 1. The flag (FL) is set as GOOD1.

Else

1. 1. 2. Go to step -2

1. 1. 3. The flag (FL) is set as BAD1.

1. 1. 4. The $\mathrm{A}_{\text {timer }}$ is $\mathrm{ON}$.

1. 1. 5. If $\mathrm{A}_{\text {timer }}$ expires, then

1. 1.5 .1. Flag is set to AWAITING1

1. 1.5. 2. If CTS \&\& ACK = True, then

1. 1. 5.2.1. Set flag as GOOD1.

1. 1. 5.2.2. Reset $A_{\text {timer }}$

Else

1. 1.5.2.3. Set flag as BAD1. End If.

1. 1.5.2.4. Double the $A_{\text {timer }}$

End if.

End if.

2. At each node,

2. 1. If $P_{S}>P_{\text {th }}$, then

Else

The flag (FL) is set as GOOD2.

End If.

The flag (FL) is set as BAD2.

2. 2. If there are $\mathrm{N}$ links in a path $\mathrm{P}$, then

End If.

$$
\text { 2. 2. } 1 . \mathrm{N}_{\max }=(\mathrm{N} / 2)+1 \text {. }
$$

2. 3. If no. of GOOD2 links $>\mathrm{N}_{\max }$, then

2. 3. 1. The path $P$ is valid and can be used for transmission.

Else

2. 3. 2. The path $P$ is invalid and marked as AWAITING2 state.

End If.

2. 4. If time $t$ in the AWAITING2 state exceeds, $t>t_{t h}$, then

End If.

2. 4. 1. The path $P$ is invalid.

Optimal Rate Adjustment Algorithm (ORAA): In prior to swapping the RTS/CTS with the receiver, a sender contends for the channel. Further, a burst of DATA/ACK pairs will be sent between the sending and receiving parties. This scenario is responsible for acclimatizing alteration in channel condition and also for re-sending corrupted packets. 


\section{J. Computer Sci., 8 (7): 1156-1164, 2012}

In our Optimal Rate Adjustment Algorithm (ORAA), the basic data rate is varied between two values namely, $R_{\min }$ and $R_{\max }$, where $R_{\min }$ is the minimum rate to which the rate can be reduced and $\mathrm{R}_{\max }$ is the maximum rate to which the rate can be increased. Suppose if the channel conditions at the two levels stated in the previous section are BAD1 and BAD2, then the current rate $\left(R_{i}\right)$ is decreased by a step value $(\lambda)$. Suppose if the channel conditions at the two levels are GOOD1 and GOOD2, then the current rate $\left(R_{i}\right)$ is increased by a step value $(\lambda)$. The steps in our rate adjustment algorithm are given under Algorithm-2. This rate adjustment is done at the receiver and applies those intended data rates in the CTS frame in such a way that the sender accepts this rate in the consequent burst of DATA frames. In addition, the estimation errors and alterations in the channel condition can be overcome by piggybacking a single bit in the ACK from the receiver to represent the optimal data rate which is possible for the next DATA frame in the burst.

\section{Algorithm-2:}

1. If the channel conditions at the two levels are BAD1 $\& \& \mathrm{BAD} 2$, then

1. 1. If $\left(R_{i}>R_{\min }\right)$ then: where $R_{i}$ is the current rate value Else

1. 1. $1 . R_{i}=R_{i}-\lambda$ : where $\lambda$ is the step value

1. 1. 2. Maintain the same rate End If.

End If.

2. If the channel conditions at the two levels are GOOD1 \& \& GOOD2, then

2. 1. If $\left(\mathrm{R}_{\mathrm{i}}<\mathrm{R}_{\max }\right)$ then

Else 2. 1. $1 . R_{i}=R_{i}+\lambda$

2. 1. 2. Maintain the same rate.

End If.

\section{RESULTS AND DISCUSSION}

\section{Experimental results:}

Simulation model and parameters: We use NS2 Network Simulator to simulate our proposed algorithm. In our simulation, the channel capacity of mobile hosts is set to the same value: 2 Mbps. In our simulation, 100 mobile nodes move in a $1500 \times 300 \mathrm{~m}$ rectangular region for $100 \mathrm{sec}$ simulation time. The Random Waypoint (RWP) model of NS2 is used for getting initial locations and movements of the nodes. We consider that every node travels independently with similar average speed. In this mobility model, a node randomly selects a destination from the physical terrain.
Table 1: Simulation settings

\begin{tabular}{ll}
\hline No. of Nodes & 100 \\
\hline Area size & $1500 \times 300$ \\
Mac & ORAA \\
Radio range & $250 \mathrm{~m}$ \\
Simulation time & $50 \mathrm{sec}$ \\
Traffic source & CBR and video \\
No. of connections & 6 \\
Packet size & 512 \\
Mobility model & Random way point $_{\text {Speed }}$ \\
Pause time & $5 \mathrm{~m} \mathrm{sec}^{-1}$ \\
Rate & $100 \mathrm{~kb}, 200 \mathrm{~kb}, \ldots . .500 \mathrm{~kb}$ \\
Error rate & $0.01,0.02, \ldots .0 .05$ \\
\hline
\end{tabular}

In our simulation, the speed is $10 \mathrm{~m} \mathrm{sec}^{-1}$. and pause time is $10 \mathrm{sec}$. The simulated traffics are Constant Bit Rate (CBR) and Variable Bit Rate (VBR) traffic. For each scenario, ten runs with different random seeds were conducted and the results were averaged.

Our simulation settings and parameters are summarized in Table 1.

Performance metrics: We compare the performance of our proposed Optimal Rate Adjustment Algorithm (ORAA) with the ADCF scheme in (Ye et al., 2009). We evaluate mainly the performance according to the following metrics.

Throughput: It is the number of packets received successfully.

Average end-to-end delay: The end-to-end-delay is averaged over all surviving data packets from the sources to the destinations.

Average packet delivery ratio: It is the ratio of the number. Of packets received successfully and the total number of packets transmitted.

Bandwidth: It is the measure of receiving bandwidth for all traffic flows.

Fairness: The fairness index is measured as the ratio of throughput of each flow and total no of flows for each flow. The performance results are presented graphically in the next section.

Based on error rate: In our initial experiment, the channel error rate varies from 0.01-0.05, with the traffic rate set at $100 \mathrm{~kb}$.

Normally, when the channel error rate is increased, the received bandwidth of all the flows will tend to decrease. As it can be seen from the Fig. 3, the bandwidth of all the flows slightly decreases, when the error rate is increased. 


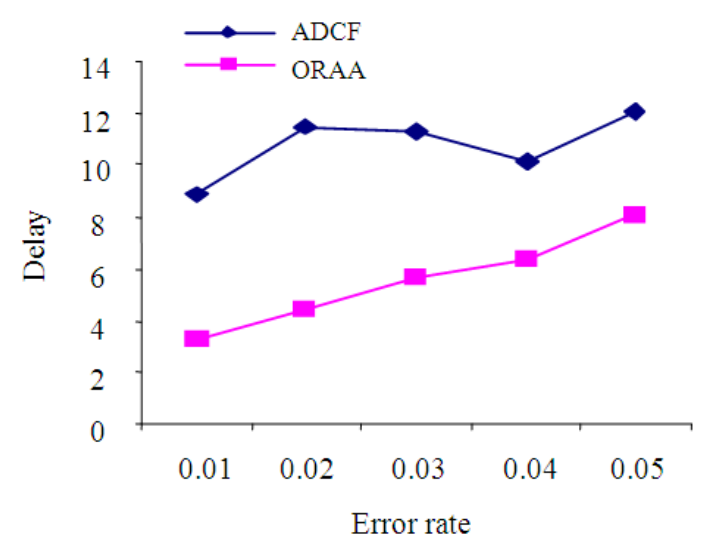

Fig. 1: Error rate Vs delay

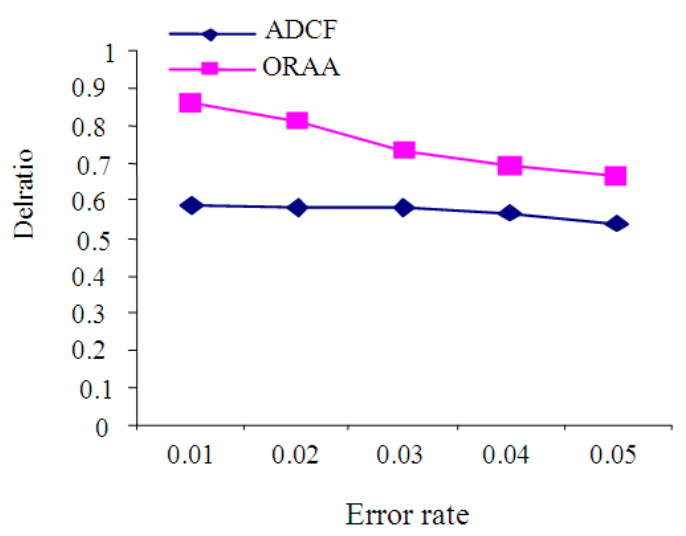

Fig. 2: Error rate Vs Delratio

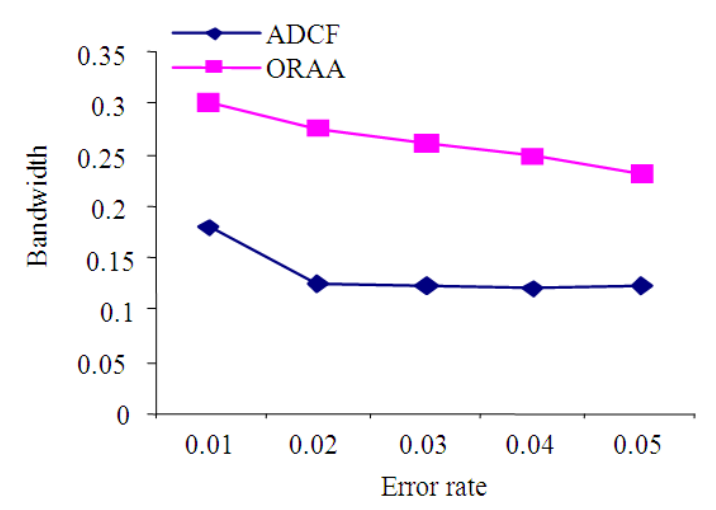

Fig. 3: Error rate Vs bandwidth

Figure 1 shows the transmission delay of both the schemes. We can find that our proposed ORAA have less delay when compared to the ADCF scheme.

From Fig. 2, it is evident that the delivery ratio of our proposed ORAA is more when compared with the ADCF scheme.

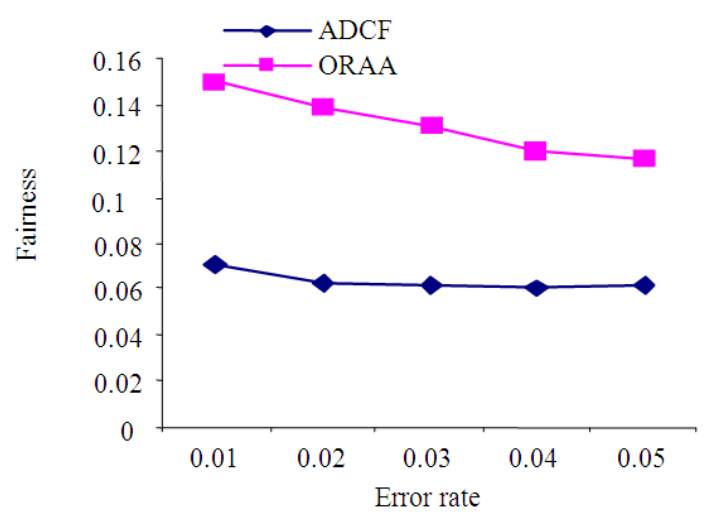

Fig. 4: Error rate Vs fairness

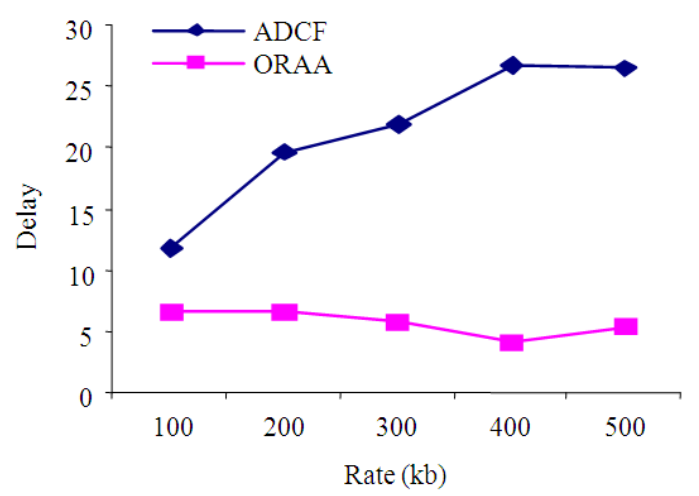

Fig. 5: Rate Vs delay

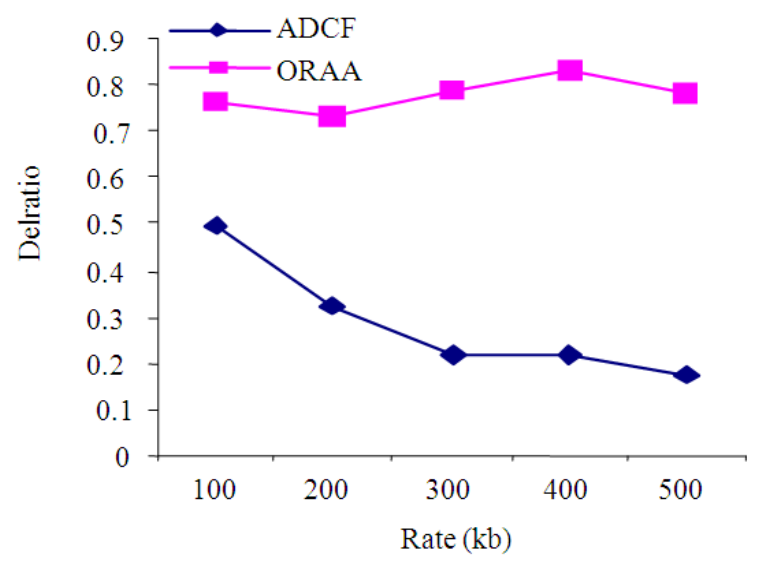

Fig. 6: Rate Vs Delratio

Figure 3 shows the bandwidth of both the schemes. It is clear that our proposed ORAA have more bandwidth when compared with the ADCF scheme. Next, we measure the fairness index. Figure 4 shows that ORAA achieves high fairness than ADCF scheme, when the error rate is increased. 


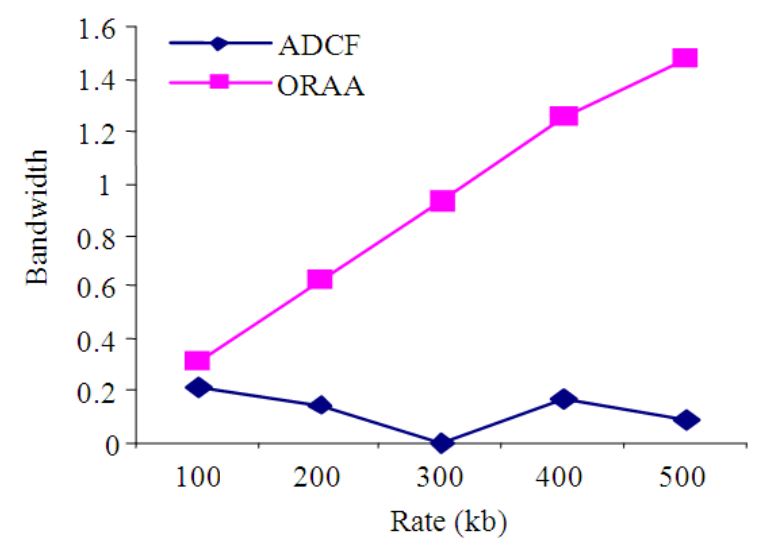

Fig. 7: Rate Vs bandwidth

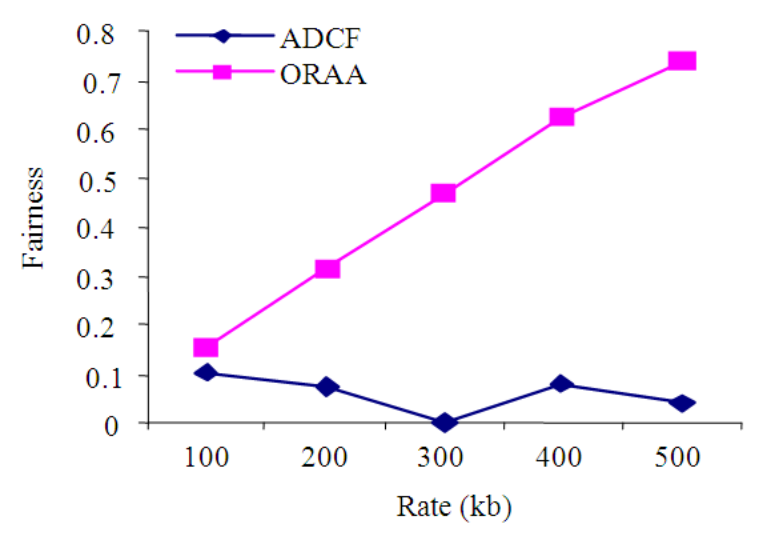

Fig. 8: Rate Vs fairness

Based on transmission rate: In our second experiment, the packet sending rate is varied from the $100-500 \mathrm{k}$, with error rate set at 0 .

Figure 5 shows the transmission delay of both the schemes. We can find that our proposed ORAA have less delay when compared to the ADCF scheme.

From Fig. 6, it is evident that the delivery ratio of our proposed ORAA is more when compared with the ADCF scheme.

Figure 7 shows the bandwidth of both the schemes. It is clear that our proposed ORAA have more bandwidth when compared with the ADCF scheme.

Next, we measure the fairness index. Figure 8 shows that ORAA achieves high fairness than ADCF scheme, when the rate is increased.

\section{CONCLUSION}

In this study we have developed an Optimal Rate Adjustment Algorithm (ORAA) based on the channel state conditions. Our channel state estimation has two levels, one at the receiver end and another at each intermediate node along the path. On the receiver side, three states namely GOOD1, BAD1 and AWAITING1 are classified based on the Packet Success rate $\left(\mathrm{P}_{\mathrm{S}}\right)$. Similarly at each intermediate node along the path, three more states namely GOOD2, BAD2 and AWAITING2 are classified based on the CTS and ACK packets. In our ORAA the rate adjustments are based on any of the above discussed channel states. Hence in ad hoc networks, where the channel conditions are dynamic, our proposed ORAA provides the accurate data rate most suitable for the current changes in the network. Simulation results show that our proposed ORAA achieves high throughput and fairness, when compared with the standard IEEE 802.11 MAC protocol.

\section{REFERENCES}

Ashraf, M., 2009. Rate adaptive channel mac' for opportunistic communication in ad hoc wireless networks. IEEE/ACM Trans. Netw.

Bandai, M., S. Maeda and T. Watanabe, 2008. Energy efficient MAC protocol with power and rate control in multi-rate ad hoc networks. Proceedings of the IEEE Vehicular Technology Conference, May 11-14, IEEE Xplore Press, Hamamatsu, pp: 66-70. DOI: 10.1109/VETECS.2008.26

Chen, Y.P., J. Zhang and A.N. Ngugi, 2007. An efficient rate-adaptive MAC for IEEE 802.11. Proceedings of the 3rd International Conference on Mobile Ad-Hoc and Sensor Networks, (MSN' 07), ACM, Heidelberg, pp: 233-243.

Hu, W., H. Yousefi'zadeh and X. Li, 2011. Load adaptive MAC: A hybrid MAC protocol for MIMO SDR MANETs. IEEE Trans. Wireless Commun., 10: 3924-3933.

Hieu, C.T. and C.S. Hong, 2010. A connection entropybased multi-rate routing protocol for mobile Ad Hoc networks. J. Comput. Sci. Eng., 4: 225-239.

Jahromizadeh, S., 2011. Joint rate control and scheduling for delay-sensitive traffic in multihop wireless networks. Proceedings of the IEEE 73rd Vehicular Technology Conference, May 15-18, IEEE Xplore Press, London, UK, pp: 1-5. DOI: 10.1109/VETECS.2011.5956645

Kherani, A., R. El-Khoury, R. El-Azouzi and E. Altman, 2008. Stability-throughput tradeoff and routing in multi-hop wireless ad hoc networks. Int. J. Comput. Telecomm. Networking, 52: 13651389. DOI: 10.1016/j.comnet.2008.01.015

Nguyen, D. and J.J. Garcia-Luna-Aceves, 2011. A practical approach to rate adaptation for multiantenna systems. Proceedings of the 19th IEEE International Conference on Network Protocols, Oct. 17-20, Santa Cruz, Canada, pp: 331-340. 
Nguyen, D., J.J. Garcia-Luna-Aceves and C. Westphal, 2011. Multi-rate adaptation with interference and congestion awareness. Proceedings of the IEEE 30th International Performance Computing and Communications Conference, Nov. 17-19, IEEE Computer Society, USA, pp. 1-8.

Ng, P.C. and S.C. Liew, 2007. Throughput Analysis of IEEE802.11 Multi-hop Ad hoc Networks" IEEE/ACM Transactions on Networking (TON), 15: 309-322. DOI: 10.1109/TNET.2007.892848

Priakanth, P. and P. Thangaraj, 2009. A channel adaptive energy efficient and fair scheduling media access control protocol for mobile adhoc networks. J. Comput. Sci., 5: 57-63. DOI: 10.3844/jcssp.2009.57.63

Pillai, M.J., M.P. Sebastian and S.D. Madhukumar, 2010. A survey of basic MAC protocols for mobile ad hoc networks. CCF College of Engineering Trivandrum.

Shrivastava, M. and and R. Sahu, 2011. Investigation on throughput of a multi hop network with identical station for random failure. Int. J. Comput. Sci. Commun., 2: 559-564.

Sadeghi, B., V. Kanodia, A. Sabharwal and E. Knightly, 2005. OAR: An opportunistic auto-rate media access protocol for ad hoc networks. Wireless Netw., 11: 39-53. DOI: 10.1007/s11276004-4745-X
Wang, K., F. Yang, Q. Zhang, D.O. Wu and Y. Xu, 2007. Distributed cooperative rate adaptation for energy efficiency in IEEE 802.11-based multi-hop networks. Proceedings of the 3rd International Conference on Quality of Service in Heterogeneous Wired/Wireless Networks, (QSHWWN' 06), ACM, USA. DOI: 10.1145/1185373.1185375

Ye, F., H. Yang, H. Yang and B. Sikdar, 2009. A distributed coordination scheme to improve the performance of IEEE 802.11 in multi-hop networks. IEEE Trans. Commun., 57: 2903-2908. DOI: 10.1109/TCOMM.2009.10.080046

Zhang, J., K. Tan, J. Zhao, H. Wu and Y. Zhang, 2008. A practical SNR-guided rate adaptation. Proceedings of the IEEE 27th Conference on Computer Communications, Apr. 13-18, IEEE Xplore Press, Beijing, pp: 2083-2091. DOI: 10.1109/INFOCOM.2008.274

Zhou, X., J. Zhao and G. Yang, 2007. Correlation based rate adaptation via insights from incomplete observations in 802.11 networks. Proceedings of the IEEE International Conference on Communications, Jun. 24-28, IEEE Xplore Press, Glasgow, pp: 4716-4721. DOI: 10.1109/ICC.2007.779 